\title{
CRYPTOCURRENCY: AN INSIGHT INTO THE MALAYSIAN REGULATORY APPROACH
}

\author{
NAZLI ISMAIL NAWANG ${ }^{*}$, IDA MADIEHA ABD GHANI AZMI ${ }^{2}$ \\ ${ }^{1}$ Faculty of Law \& International Relations, Universiti Sultan Zainal Abidin, 21300 Terengganu, Malaysia. \\ inazli@unisza.edu.my* (Nazli Ismail Nawang) \\ ${ }^{2}$ Ahmad Ibrahim Kulliyyah of Laws, International Islamic University Malaysia, 53100 Gombak, Selangor Malaysia.
}

\begin{abstract}
Cryptocurrency like Bitcoin, Ethereum and many others are closely affiliated with the financial technology (fintech) industry. These digital or virtual currencies are encrypted using cryptography and distributed on public ledger (blockchain) across decentralised peer-to-peer (P2P) networks. Since cryptocurrencies are issued by private entities and technically beyond the controls of any states, they may be exploited by criminals for illicit purposes such as money laundering, terrorism financing and many others. Thus, this article will examine whether cryptocurrency amounts to a new form of money under the existing rules on financial services and analyse how it should be regulated. This study is a qualitative research that adopts pure legal research methodology by analysing existing laws on national currency and digital currency. Further, the study analysed other secondary sources including academic books, journal articles, conference papers, and other materials in newspapers or reputable websites. The output of the research is expected to provide an overview on the regulatory framework for cryptocurrency in Malaysia. In conclusion, the government has favoured a minimalist approach so as not to stiffen the innovation and future development of cryptocurrency in the country.
\end{abstract}

Keywords: Bitcoin, Cryptocurrency, Digital Currency, Malaysia, Regulatory Framework

Article Received: 18 October 2020, Revised: 3 November 2020, Accepted: 24 December 2020

\section{Introduction}

Cryptocurrency like Bitcoin, Ethereum, Ripple and many others have become popular over the last few years, in particular among the players in the financial technology (fintech) industry. The first cryptocurrency, Bitcoin, was introduced in 2009 by Satoshi Nakamoto (a pseudonymous person or group of persons) subsequent to the online publication of a paper entitled 'Bitcoin: A Peer-to-Peer Electronic Cash System in 2008. Since then, a number of cryptocurrencies have been developed and as at April 2020, there are a total of 5,315 types of cryptocurrencies worldwide with a market capitalisation of almost USD 200 billion. Despite the fact that currently they are only 788 cryptocurrencies that are worth more than USD 1 million market capitalisation, it is arguably impossible for such words to be unheard of or unknown in this digital era.

With regard to its nature, cryptocurrency is in its purest form generated in intangible digital codes and therefore does not require any physical resources like metal, paper or plastic to generate. These digital or virtual currencies, which are issued not by traditional financial institutions but rather by private entities, run on universal peer-topeer (P2P) networks of computers and are fully distributed on universal public ledger, known as blockchain. Further, cryptocurrency enables anonymous transactions as the addresses of cryptocurrencies consist of only an alphanumeric sequence of characters and not true identities of the transacting parties (Jonathan, 2015).

Nonetheless, cryptocurrency like Bitcoin is not entirely an anonymous currency. This is because all trails of transactional records between the parties are publicly shared and can possibly be traced. Thus, the real-world identities of the participants may be revealed by any skilled persons (Lam, 2015). For that reason, Bitcoin and other cryptocurrencies have been described as 'partially anonymous' (Reuben, 2011), whilst some scholars asserted that they are rather pseudonymous and not completely anonymous 
(Edward, 2015). In relation to this, it was claimed that this common misconception is due to the lack of understanding of the new technology used for the creation of cryptocurrencies (Jerry, 2013).

The decentralised and anonymous (or pseudonymous) nature of cryptocurrency have enabled and facilitated criminals in engaging in a number of illegal activities such as money laundering, financing of terrorism and many others. The best illustration is the revelation of Silk Road, a black-market website that accepted Bitcoin for the trade of illegal drugs (Daniel, 2014). Such scenario has undoubtedly raised a legal concern over the monitoring and regulating of cryptocurrency.

As to the position in Malaysia, the Parliament has enacted specific provision to deal with anonymous offenders in the cyber world (Nazli, 2017). Further, offensive content on the Internet may be adequately tackled by the existing cyber laws (Ammar, 2019). Though it was argued that no changes are necessary to the Penal Code in dealing with criminal cases involving digital currencies as reported incidents are very scarce (Razinah, 2019), the Central Bank of Malaysia (BNM) has closely monitored the adoption and widespread use of cryptocurrency in the country. Since the potential use of cryptocurrency for illegal activities is also a major concern for the law enforcement authorities, this article will therefore examine the legal status of this new form of currency under the existing rules on financial services and analyse how cryptocurrency should be regulated and finally propose some recommendations and solution to the issue.

\section{Overview of Cryptocurrency}

The term 'cryptocurrency', which is a portmanteau of two words; 'cryptography' and 'currency', may be understood in simple terms to refer to digital or virtual currencies that are encrypted using cryptography. The word 'cryptography' originates from the Greek word 'krypto' which literally means 'hidden' (Daniel, 2002). Cryptography has been defined as the art and science of keeping information secret by using a code or cipher and it has been used throughout history mainly to keep communications private (Terrence, 1998). At present, cryptography has been adopted to secure transfers of information and payment transactions in the electronic environment.

The importance of cryptography has been highlighted by the Organisation for Economic Cooperation and Development (OECD) in the OECD Guidelines for Cryptography Policy (OECD 1997) which stipulates that:

"Cryptography can be an effective tool for the secure use of information technology by ensuring confidentiality, integrity and availability of data and by providing authentication and nonrepudiation mechanisms for that data, it is an important component of secure information and communications networks and systems ... the failure to utilize cryptographic methods can adversely affect the protection of privacy, intellectual property, business and financial information, public safety and national security and the operation of electronic commerce because data and communications may be inadequately protected from unauthorized access, alteration and improper use, and therefore users may not trust information and communications systems, networks and infrastructures".

\section{Interpretation of Cryptocurrency}

As to the interpretation of the word 'cryptocurrency', there is no universally agreed definition of cryptocurrency. The word is elusive and varied according to the understanding and interpretation of scholars, financial experts, fintech industry players and members of the public at large. The followings are the interpretations of the term 'cryptocurrency' as laid down by among the most established financial organisations in the world, legal scholars and under the statutes in Malaysia.

\subsection{European Central Bank (ECB)}

The European Central Bank (ECB) has classified cryptocurrencies as a subset of virtual currencies. In a report on Virtual Currency Schemes of 2012, 
the ECB defined virtual currencies as "a type of unregulated, digital money, which is issued and usually controlled by its developers, and used and accepted among the members of a specific virtual community". In the subsequent report of 2015 titled Virtual Currency Schemes - A Further Analysis, the ECB has expressly stated that it does not consider virtual currency like Bitcoin as full forms of money as it is not legally regarded as neither money nor currency. The ECB has also updated the definition of virtual currencies as "digital representations of value, not issued by a central bank, credit institution or e-money institution, which in some circumstances can be used as an alternative to money".

\subsection{International Monetary Fund (IMF).}

The International Monetary Fund (IMF) has categorised cryptocurrencies as a subset of virtual currencies which are then interpreted as "digital representations of value, issued by private developers and denominated in their own unit of account". It is further elaborated that virtual currencies "can be obtained, stored, accessed, and transacted electronically, and can be used for a variety of purposes, as long as the transacting parties agree to use them" (Dong, 2016).

\subsection{World Bank}

The World Bank has classified cryptocurrencies as "a subset of digital currencies that rely on cryptographic techniques to achieve consensus". Whilst the term digital currencies are defined as "digital representations of value that are denominated in their own unit of account, distinct from e-money, which is simply a digital payment mechanism, representing and denominated in fiat money" (Harish, 2017).

In short, it is submitted that these definitions have in general characterised cryptocurrencies as a form of decentralised private payment mechanisms that are digitally or electronically represented in their own denominations. Cryptocurrencies can be used as an alternative to money between the consenting parties, even though they are not legally accepted as legal tender or money. It is observed that out of the aforesaid descriptions, only the World Bank has forwarded a clearer definition by highlighting the utilisation of cryptographic algorithms for cryptocurrency.

Apart from the aforesaid institutional interpretations, a number of scholars have provided some assistance in detailing the distinctive features of cryptocurrencies. Cryptocurrencies have been described as a subset of virtual currencies that are issued by private entities with intrinsic economic value that is comparable to legal tender issued by governments (Jonathan, 2015). A more specific narrative of cryptocurrencies is expounded whereby they are regarded as a form of digital currency that relies on encryption techniques to regulate the generation of its units and to verify the transfer of funds without the involvement of any financial institutions (Sonny, 2017). Similar explanation is adopted as cryptocurrencies is regarded as a digital currency that are based on encryption techniques to control the creation of such currencies and to validate the execution of payment transactions on a decentralised network (Benjamin, 2019).

\subsection{Statutory Meaning of Cryptocurrency}

As to the statutory interpretation of 'cryptocurrency' in Malaysia, reference has to be made to the statutes under the purview of BNM, namely the Financial Services Act 2013 (FSA) and the Islamic Financial Services Act 2013 (IFSA). The FSA is an extensive legislation that consolidates various legislations pertaining to banking, investment banking, insurance and payment systems businesses and the oversight of the money market and foreign exchange administration in Malaysia. The FSA has also combined and repealed the Banking and Financial Institutions Act 1989, the Insurance Act 1996, the Exchange Control Act 1953 and the Payment Systems Act 2003. With regard to the IFSA, it has substituted and repealed the Islamic Banking Act 1983 and the Takaful Act 1984. Further, the study has also examined relevant subsidiary legislation that may be applicable to cryptocurrency such as the Anti-Money Laundering and Counter 
Financing of Terrorism Policy for Digital Currencies (Sector 6), Capital Markets and Services (Prescription of Securities) (Digital Currency and Digital Token) Order 2019 and many others.

According to the existing statutes, neither the words 'cryptocurrency' nor 'virtual or digital currency' are defined in the FSA and IFSA. The closest inference may be referred to the interpretation of 'electronic money'. Section 2(1) of the FSA defines electronic money as:

"any payment instrument, whether tangible or intangible that -

(a) stores funds electronically in exchange of funds paid to the issuer; and

(b) is able to be used as a means of making payment to any person other than the issuer".

Almost similar interpretation of electronic money is stipulated in section 2(1) of the IFSA which refers electronic money as:

"any Islamic payment instrument, whether tangible or intangible, that -

(a) stores funds electronically in exchange of funds paid to the issuer; and

(b) is able to be used as a means of making payment to any person other than the issuer".

From the aforesaid interpretations, it is conceivable that cryptocurrency appears to possess certain functions of electronic money, in particular as a store of value and medium of exchange. Nonetheless, it was cautiously underlined that electronic money is totally different from cryptocurrency or virtual currency as electronic money represents fiat money or legal tender that is kept electronically in digital accounts and is subject to the regulatory controls of the BNM (Zalina, 2016).

Apart from that, section 63 of the Central Bank of Malaysia Act 2009 has explicitly defined legal tender in Malaysia as currency notes and coins issued by BNM. The exclusive authority of BNM in issuing legal tender is further stressed in section
10 of the Currency Act 2020. ${ }^{1}$ As such, the concept of electronic money and cryptocurrencies under the Malaysian law is not legally comparable as the two are distinctively distinguishable since the former equates legal tender whilst the latter is not recognised as a legal tender as it is issued by other private entities.

4. The Development and Usage of Cryptocurrency in Malaysia

Cryptocurrency has marked its entry into the financial limelight after the unfortunate occurrence of the Global Financial Crisis in 2008 and the subsequent introduction of Bitcoin on the Internet by Satoshi Nakamoto a year later (Georgios, 2019). Since then, cryptocurrency has been gaining popularity and been taken up by investors and traders across the globe including Malaysia. Nonetheless, due to the lack of authoritative statistics and official records on the acceptance and usage of cryptocurrencies in Malaysia, it is believed that based on the postings in BitcoinMalaysia.com, Bitcoin has been traded in the country since 2012 .

In March 2014, it was estimated that there were around 2,000 Bitcoin users in Malaysia (Christina, 2019) and 26 Malaysian based merchants, mostly in the Klang Valley, have accepted Bitcoin and other types of cryptocurrencies including Dodge and Litecoin for payments of their goods and services in 2017 (Colbert, 2017). With regard to the value of cryptocurrency transactions in Malaysia, BNM reported in December 2017 that an average of RM 75 million were traded each month on four digital currencies exchanges; namely Luno, Coinhako, XBit Asia and PinkExchange (Intan, 2017).

Notwithstanding the aforesaid figures, the acceptance of cryptocurrency among the people in this country is still low and is at its infancy stage as compared to the global reception of these currencies (Sonny, 2017). A number of potential

\footnotetext{
${ }^{1}$ The Digital Currency Act 2020 (Act 827) was enacted on 14 February 2020 and published in Gazette on 28 February 2020. However, until today it has yet to come into operation.
} 
risks may be associated with the slow take-up of cryptocurrencies by the Malaysian consumers including "loss or theft, fraud or unauthorised use, transaction processing errors, failure of a wallet or exchange and inadequate disclosure" (Zalina, 2016). Consequently, the government has come up with a warm approach in regulating cryptocurrencies in Malaysia (Durgha, 2018). As such, it is pertinent to analyse the regulatory approach that has been adopted to govern Bitcoin and other virtual currencies in the country.

5. The Malaysian Legal Framework on Cryptocurrency

The supervision and monitoring of cryptocurrency in Malaysia can be first traced to the year 2014 when BNM issued an official statement declaring that Bitcoin, the most famous cryptocurrency, is not recognised as legal tender in Malaysia and is not subjected to its regulatory control. This is in parallel with section 63 of the Central Bank of Malaysia Act 2009 which provides that only currency notes and coins issued by BNM are regarded as legal tender. However, the statement is not to be construed as the trading of Bitcoin or other types of cryptocurrencies is completely prohibited. The statement by BNM is ought to be construed as an advice to the public to be cautious of the risks associated with the usage of cryptocurrency. Therefore, any individual traders or investors may still engage in transacting any types of cryptocurrencies in the country though the statement impliedly indicated that the BNM had taken a hard stance towards cryptocurrencies in their early days in the country.

At the end of 2017, BNM appeared to have taken a different approach in regulating cryptocurrency exchanges in Malaysia when such currencies are regarded to be the "new norm" and that BNM "cannot be oblivious to these developments" (Risen, 2017). In February 2018, BNM has issued the official cryptocurrency regulation in Malaysia under the policy paper "Anti-Money Laundering and Counter Financing of Terrorism Policy for Digital Currencies (Sector 6)". Pursuant to the policy paper, reporting institutions under Sector 6 that operate digital currency exchanges will be subject to obligations under the Anti-Money Laundering, Anti-Terrorism Financing and Proceeds of Unlawful Activities Act 2001 (AMLA). And pursuant to Paragraph 4.2 of Sector 6 , the obligations shall apply to such institutions even though they do not have any physical presence in Malaysia.

Digital currency exchanges are also mandated to conduct adequate risk assessment on their customers in order to the prevent the potential use of cryptocurrencies for money laundering and financing of terrorism, while increasing the transparency of digital currency activities in Malaysia. Apart from that, the digital currency exchanges as reporting institutions are required to conduct Customer Due Diligence or better known as Know-Your-Customer (KYC) that are not entirely different from what licensed entities regulated by Bank Negara Malaysia are subject to. Nonetheless, the imposition of these obligations on the digital currency exchanges or companies that offer cryptocurrencies to the public in Malaysia does not mean that they are allowed to portray themselves as licensed entities under BNM.

In recognising certain digital currencies and digital tokens that have met the prescribed condition as 'securities' under the regulation of the Securities Commission of Malaysia (SC), the Capital Markets and Services (Prescription of Securities) (Digital Currency and Digital Token) Order 2019 was introduced in January 2019. Regulation 2 of Order 2019 has defined digital currency as "a digital representation of value which is recorded on a distributed digital ledger whether cryptographically-secured or otherwise, that functions as a medium of exchange and is interchangeable with any money, including through the crediting or debiting of an account". Whilst the definition of digital token is provided in the same regulation as "a digital representation which is recorded on a distributed digital ledger whether cryptographically-secured or otherwise". Almost similar interpretation of digital currency is 
provided in Paragraph 6.2 of Sector 6, but it further excludes electronic money as defined in the FSA and IFSA. Thus, it is submitted that the lack of statutory interpretation of the word 'cryptocurrency' in the FSA and IFSA has now been remedied with the definitions of digital currency and digital token in Order 2019.

Pursuant to Regulation 4 of Order 2019, digital currencies and tokens are recognised as securities and therefore, issuers of such currencies or tokens i.e. Digital Assets Exchange (DAX) operators are required to apply for a Capital Markets Services License under Section 58 of the Capital Markets and Services Act 2007 (CMSA) in order to carry out 'regulated activities' as specified in Schedule 2 of the CMSA. Failure to comply with the prescribed requirement will expose DAX operators to a fine of up to RM10 million and / or up to 10 years in prison. With the coming into force of Order 2019 on 15 January 2019, the SC has issued revised Guidelines on Recognized Markets on 31 January 2019 for DAX operators to be registered as a recognized market operator (RMO). According to the revised guidelines, recognised market operators must protect clients' interests by keeping latest records of investors as well as the money and digital assets held, segregating trust accounts that receive money from and pay to licensed financial institutions and making arrangements to protect clients against risks, loss, and also theft. Currently, three RMOs; namely Luno Malaysia Sdn Bhd, SINEGY Technologies (M) Sdn Bhd and Tokenize Technology (M) Sdn Bhd, have been registered to establish and operate digital asset exchanges in Malaysia.

The legal status of cryptocurrency has finally been tested in courts in the unreported case of Luno Pte Ltd \& Anor v Robert Ong Thien Cheng. ${ }^{2}$ In this case, the plaintiffs operated an exchange of digital currencies and the defendant was one of their customers. Due to some technical glitch, the

\footnotetext{
${ }^{2}$ Sessions Court Civil Suit No. BA-B52NCVC-389-12/2017 (Unreported).
}

plaintiffs had mistakenly transferred an additional of 11.3 Bitcoins, instead of only 11.3 Bitcoins and thereby totalling 22.6 Bitcoins to the defendant's e-wallet account with a third party. Upon realising the double transfers, the plaintiffs requested the defendant to return the additional of 11.3 Bitcoins, but the request was ignored by the defendant. The plaintiffs then sued the defendant for the recovery of 11.3 Bitcoins or its equivalent value.

At the court of the first instance, the defendant was ordered to return 11.3 Bitcoins or its equivalent value in Ringgit Malaysia to the plaintiffs. On appeal, the Sessions Court judge's sentence was upheld, and the appeal was dismissed by the High Court. ${ }^{3}$ Among the important principles derived from the first case on cryptocurrency in the country is that although Bitcoin or other digital currency is not regarded as legal tender in Malaysia, it has been regarded as a form of commodity because real money is used to purchase the cryptocurrency. Further, it was ruled that cryptocurrency trading is not illegal as it has been regulated by the SC. Regardless of this current position, the case in now pending at the Court of Appeal.

\section{Conclusion}

This study primarily aims at exploring the development of cryptocurrency in Malaysia, the legal position of such currency under the existing laws and the regulatory approach that has been adopted to regulate cryptocurrency in the country. A close scrutiny of the historical development of cryptocurrencies since 2012 until today revealed that huge potentials of the currency have yet to be exploited by the Malaysian public. A number of factors may have contributed to the slow take-up of these cryptocurrencies and most of them centred around uncertainty relating to the security and consumer protection issues.

As to the legal position of cryptocurrency, this issue has finally been resolved as digital currency has been statutorily regarded as a form of digital

\footnotetext{
${ }^{3}$ Shah Alam High Court Civil Appeal No. 12BNCVC-91-10/2018.
} 
asset which is under the purview of the SC. Even though cryptocurrency has not been accorded the status of legal tender by the BNM, any financial transactions involving cryptocurrency are unlawful as evidenced by the judgment in the case of Luno Pte Ltd \& Anor $v$ Robert Ong Thien Cheng. Hence, cryptocurrency like Bitcoin and Ethereum may be used as a mode of payment to purchase goods and services offered by merchants that are willing to accept them as a form of payment or be traded on recognised digital asset exchange (DAX) platforms. With regard to the regulatory approach that has at present been adopted by the government to govern cryptocurrency, it is submitted that the government has favoured a minimalist approach so as not to stiffen the innovation and future development of cryptocurrency in the country.

\section{Acknowledgments}

The researchers wish to thank the Ministry of Higher Education of Malaysia for providing the Postdoctoral Scholarship (KPM(BS)75091211XXXX) that has enabled this research and publication.

\section{References}

[1] Amor, D. (2002). The e-business (r) evolution: living and working in an interconnected world. Prentice Hall PTR.

[2] Ann Daniels, (2014). Bitcoin and Illegal Activity: Silk Road Defendants Pled Guilty on September 4, 2014, UIC Journal of Privacy \& Technology Law. available at https://jitpl.jmls.uic.edu/2014/09/13/bitcoi n-and-illegal-activity-silk-road-defendantspled-guilty-on-september-4-2014/

[3] Brito, J., \& Castillo, A. (2013). Bitcoin: A primer for policymakers. Mercatus Center at George Mason University.

[4] Christina C. (2014). Be Wary of Virtual Money, M'sians Told, The Star Online. Retrieved 15 April 2020 available at https://www.thestar.com.my/news/nation/2 014/03/30/be-wary-of-virtual-money- msians-told-bitcoin-is-not-recognised-aslegal-tender-here-stresses-bank-ne

[5] Colbert, (2017). List of Bitcoin Accepting Merchants in Malaysia. Retrieved 15 April 2020 available

at

https://www.bitcoinmalaysia.com/2014/07/ 20/list-of-bitcoin-accepting-merchants-inmalaysia

[6] Dimitropoulos, G. (2019). Global Currencies and Domestic Regulation: Embedding through Enabling?.

[7] Durgha, M. (2018). A Study on Rising Effects of Cryptocurrency in the Regulations of Malaysian Legal System, International Journal of Business, Economics and Law, vol. 15, no. 4, pp. 35-41.

[8] Geva, B. (2019). Cryptocurrencies and the Evolution of Banking, Money and Payments. Cryptoassets Legal, Regulatory and Monetary Perspectives (Oxford University Press, 2019) pp, 11-38.

[9] Grinberg, R. (2011). Bitcoin: An innovative alternative digital currency. Hastings Science \& Technology Law Journal, 4, 160.

[10] He, D., Habermeier, K. F., Leckow, R. B., Haksar, V., Almeida, Y., Kashima, M., ... \& Yepes, C. V. (2016). Virtual currencies and beyond: initial considerations.

[11] IEEE.Ammar Abdullah Saeed Mohammed \& Nazli Ismail Nawang, (2019). Offensive Content on The Internet: The Malaysian Legal Approach, International Journal of Innovation, vol. 5, no. 2, pp. 367-377.

[12] Intan Farhana Zainul, (2017). BNM: RM75m Cryptocurrencies Transactions Each Month, The Star Online, December 16. Retrieved 15 April 2020 available at https://www.thestar.com.my/business/busi ness-news/2017/12/16/digital-currenciesworth-rm75m-transacted-each-month

[13] Lam Pak Nian and David Lee Kuo Chuen. (2015). Introduction to Bitcoin. Edited 
David Le Kuo Chuen, Elsevier, San Diego, pp. 5-30.

[14] Lim, J. W. (2015). A facilitative model for cryptocurrency regulation in Singapore. In Handbook of Digital Currency (pp. 361381). Academic Press. https://doi.org/10.1016/B978-0-12802117-0.00018-7

[15] Murphy, E. V., Murphy, M. M., \& Seitzinger, M. V. (2015, October). Bitcoin: Questions, answers, and analysis of legal issues. Library of Congress, Congressional Research Service.

[16] Natarajan, H., Krause, S., \& Gradstein, H. (2017). Distributed ledger technology and blockchain. World Bank.

[17] Nawang, N. I. (2017, March). Combating anonymous offenders in the cyberspace: An overview of the legal approach in Malaysia. In 2017 2nd International Conference on Anti-Cyber Crimes $(I C A C C)$ (pp. 13-18).

https://doi.org/10.1109/Anti-

Cybercrime.2017.7905255

[18] Risen Jayaseelen and Cecilia Kok, (2017). Bank Negara Reins in Digital Currencies, The Star Online, November 23, Retrieved 15 May 2020 available at https://www.thestar.com.my/business/busi ness-news/2017/11/23/bank-negara-reinsin-digital-currencies/

[19] Stender, J. T. (1998). Too many secrets: Challenges to the control of strong crypto and the national security perspective. Case W. Res. J. Int'l L., 30, 287.

[20] Zain, N. R. B. M., Ali, E. R. A. E., Abideen, A., \& Rahman, H. A. (2019). Smart contract in blockchain: An exploration of legal framework in Malaysia. Intellectual Discourse, 27(2), 595-617.

[21] Zalina Muhamed Zahudi and Radin Ariff Taquiddin Radin Amir. (2016). Regulation of Virtual Currencies: Mitigating the Risks and Challenges Involved, Journal of Islamic Finance, vol. 5, no. 1, pp. 63-73.

[22] Zulhuda, S., \& binti Sayuti, A. (2017). Whither Policing Cryptocurrency in Malaysia?. IIUM Law Journal, 25(2), 179196. 